Chimia 45 (1991) 282-284

(c) Schweiz. Chemiker-Verband; ISSN 0009-4293

\title{
Jenseits des Sichtbaren, Farbstoffe zweiter Ordnung [1]
}

\author{
József Kelemen*
}

Von allen NIR-absorbierenden Farbstoffen sind jene aus der Küpengamme die rätselhaftesten. Dies hängt damit zusammen, dass sie einst grosse Bedeutung als militärische NIR-Tarnfarbstoffe erlangten und strengster Geheimhaltung unterlagen. Ein brauchbares Schrifttum existiert kaum Es ist anzunehmen, dass diese Farbstoffe nach phänomenologischen Gesichtspunkten gesucht, gefunden und nach Analogieschlüssen weiterentwickelt wurden. Die Natur ihrer NIR-Absorption ist unerforscht, das Wesen ihres NIR-Chromophors ist unbekannt. In diesem Beitrag versuchen wir diese Lücke wenigstens in den Ansätzen zu füllen.

\section{Umfang der NIR-Absorption von Kü- penfarbstoffen}

Remissionsspektroskopische Untersuchungen an Färbungen einer repräsentativen Sammlung von Küpenfarbstoffen ergaben grosse Unterschiede in der spektralen Lage der NIR-Banden und in ihrer Absorptionsstärke. Während einfache Anthrachinonderivate und indigoide Farbstoffe in dem Farbtonbereich von gelb bis rot praktisch keine NIR-Absorption aufweisen, zeigen solche mit tiefem Farbton (violett, blau, grün, oliv, braun und schwarz) ausgeprägte Absorption in dem Wellenlängenbereich von 750 bis $1500 \mathrm{~nm}$. Als Stellvertreter dieser Klasse haben wir C. I. Vat Black 30 eingehender untersucht. Er gehört zu den wirksameren NIR-Absorbern. Aus den KubelkaMunk-Spektren [2] seiner Färbungen konnten zwei Absorptionsbanden bei 1260 und $1040 \mathrm{~nm}$ identifiziert und die Intensitäten dieser Absorptionen geschätzt werden. Der molare Extinktionskoeffizient beträgt etwa 2000-3000 für die erste, und 3000-5000 $\mathrm{M}^{-1} \mathrm{~cm}^{-1}$ für die zweite Bande. Diese Daten schliessen sowohl Schwingungsübergänge, die in diesem Spektralbereich erwartet werden ( $\varepsilon \sim 0.1-10$ für erste Oberton- und Kombinations- und $<1$ für zweite Obertonschwingungen) wie auch $n, \pi^{*}$-Absorption $(\varepsilon \sim 10-1000)$ aus. Obwohl die chemische Struktur des eingesetzten Farbstoffes zwei-

*Korrespondenz: Dr. J. Kelemen Departement Forschung und Entwicklung Division Farbstoffe und Chemikalien Ciba-Geigy AG, Werk Klybeck C.H-4002 Base fellos korrekt ist, sind Spektrum und Struktur miteinander nicht vereinbar. Eine ähnliche Situation wurde auch bei anderen NIR absorbierenden Küpenfarbstoffen gefunden. Eine Sichtung der theoretisch möglichen Mechanismen soll hier das Auffinden des NIR-Chromophors, von dem nur sein $\pi, \pi^{*}$ Charakter gewiss ist, erleichtern.

\section{Strategien für NIR-Absorption}

In der MO-Theorie wird Lichtabsorption als Übergang eines Elektrons aus einem voll- oder halbbesetzten in ein leeres oder halbleeres MO beschrieben. Die spektrale Lage der Absorptionsbande ist durch die Energiedifferenz (Gap) dieser beiden $\mathrm{Or}$ bitale bestimmt. Die kleinste, für die $A b$ sorption nutzbare Energiedifferenz, welche die Lage der langwelligen Absorptionsbande bestimmt, ist jene zwischen HOMO und LUMO und beträgt bei Farbstoffen zwi- schen 2-4 eV. Um NIR-Absorption zu erreichen, wird eine, für den spektralen Übergang verwertbare, Energiedifferenz von $\sim 1$ $\mathrm{eV}$ benötigt. Ein Farbstoff 1. Ordnung weist eine ganze Anzahl solcher Gaps auf. Diese sind entweder zwischen den vollbesetzten bindenden oder zwischen den leeren antibindenden MOs zu finden. Sie können jedoch für die Lichtabsorption nicht ohne weiteres genutzt werden, weil entweder beide Molekülorbitale doppelt besetzt oder beide leer sind. Fig. la veranschaulicht diese Situation schematisch für einen typischen Farbstoff. Von diesem Bild ausgehend gibt es verschiedene Möglichkeiten, um die niedrigste Absorptionsbande ins NIR zu verschieben.

\section{Auf molekularer Ebene:}

i) Durch chemisch-konstitutionelle Strukturvariation, welche den HOMO-LUMO Abstand verkleinern (Fig. 1b).

Als solche sind die Erweiterung des $\pi$ Systems, die Einführung nicht-alternierender Strukturelemente und die «push-pull»Substitution zu nennen. Solche Systeme sind diamagnetisch mit einem Singulett Elektronengrundzustand. Dies sind die klassischen Methoden. Nach einer anderen Betrachtungsweise entstehen solche langwellig absorbierende Spezies durch die Aufweitung der «Beinahe-Entartung» biradikaloider Strukturen [3].

ii) Durch Änderung der Elektronenkonfiguration (Besetzungsschema) des Systems dadurch, dass die geschlossene Elektronen-

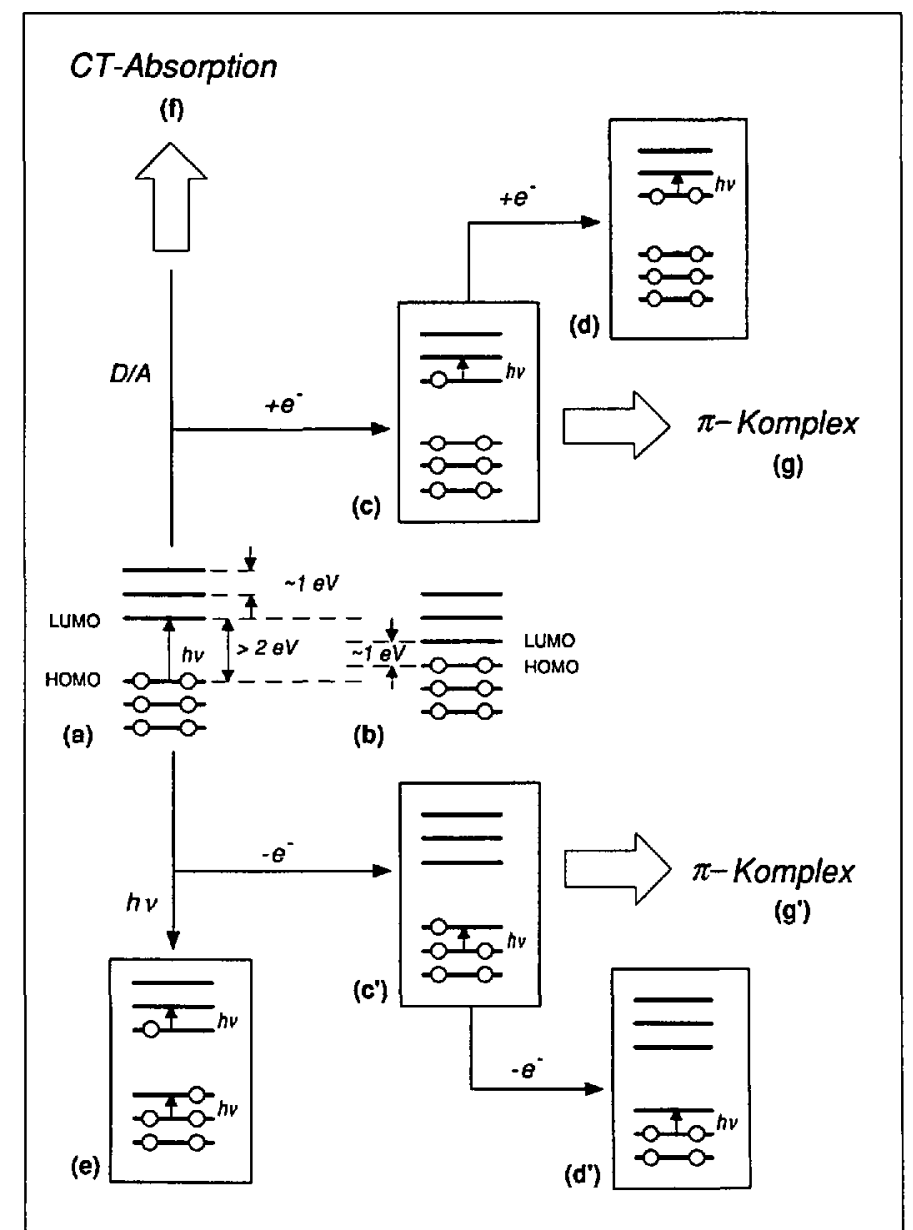

Fig. 1. NIR-Absorptionsmechanismen in Übersicht (s. Text) 
schale erweitert, einengt oder aufgebrochen wird.

Solche Massnahmen legen eine oder mehrere kleine Gaps für Elektronenübergänge frei und führen zu einer ungewöhnlich langwelligen Absorption. Hier sind die $1 \mathrm{e}^{-}$-Reduktion, d.h. das Zufügen eines Elektrons zum LUMO (Fig. Ic), die $1 \mathrm{e}^{-}$-Oxydation, d.h. der Entzug eines Elektrons aus dem HOMO (Fig. IC'), die MehrelektronenRedoxprozesse (Fig. Id, d'), und das optische Pumpen (Fig. le), d.h. die Anhebung eines Elektrons auf ein höheres Niveau [4], zu erwähnen. Die Einelektronenprozesse führen zu paramagnetischen Spezies (Radikale) mit einem Dublett-Elektronengrundzustand.

Auf zwischenmolekularer Ebene:

iii) Zwischen zwei Komponenten mit abgeschlossener Elektronenschale (Fig. If).

Die Kombination eines diamagnetischen Donors mit niedrigem Ionisierungspotential (d.h. mit hochliegendem HOMO) mit einem diamagnetischen Akzeptor mit hoher Elektronenaffinität (tiefliegendem LUMO) führt zu einer langwelligen CT-Absorption, die gewöhnlich von niedriger Intensität ist (Fig. If). Die CT-Absorption von Tetracyanoethylen/Perylen liegt bereits im NIR (920 nm) [5]. Die Chinhydrone sind diamagnetische Donor-Akzeptor-Molekülkomplexe zwischen einem Hydrochinon (Donor) und einem Chinon (Akzeptor), die auch aus der Disproportionierung monoprotonierter Semichinonradikale entstehen. Ihre CT-Absorption ist sehr langwellig ( $\sim 550 \mathrm{~nm}$ [6]).

iv) Zwischen zwei Komponenten mit offener Elektronenschale (Radikale).

Die $\pi$-Komplexe, die aus freien Radikalen oder aus Radikal-Salzen gebildet werden, führen oft zur NIR-Absorption (Fig. $\left.l g, g^{\prime}\right)$. Die $\pi$-Komplexe selber sind diamagnetisch, stehen aber mit den weniger langwellig absorbierenden radikalischen Spezies im Gleichgewicht, wie von den Wursterschen Salzen und vom $\mathrm{N}$-Ethylphenazin her bekannt ist [7].

v) Zwischen zwei Komponenten ( $\mathrm{Li}$ ganden), deren Elektronenschalen gemischter Natur sind: eine offene und eine geschlossene Schale.

Metallkomplexe mit einer starken $\pi$ Wechselwirkung zwischen den Liganden, die sich in unterschiedlichen Redoxstufen befinden, gehören hierzu, wie die Lanthanid(III)-Komplexe mit Phthalocyanin-und Octaethylporphyrin-Liganden [8], die NIR-Banden bis über $1400 \mathrm{~nm}$ aufweisen.

Die erste Strategie führt zu diamagnetischen NIR-Farbstoffen mit geschlossener Elektronenschale. Die resultierenden Spezies sind in der Regel thermodynamisch stabil und chemisch beständig. Darin liegt die grosse Popularität dieser Strategie. Für diesen finden wir in den chemischen Strukturen der untersuchten Küpenfarbstoffe keinen Anhaltspunkt. Die Änderung der Elektronenkonfiguration (ii) führt zu Ver- bindungen mit offener Elektronenschale (paramagnetische Radikale) oder zu mehrfach geladenen Ionen. Die resultierenden Verbindungen absorbieren gelegentlich im NIR, sie gelten aber als zu reaktiv, zu anfällig für Disproportionierung und Dimerisierung oder sie sind von transienter Natur. Die CTAbsorptionen (iii) sind nur in echten DonorAkzeptor Komplexen stark, sonst sind sie relativ schwach wegen der schlechten Überlappung der $\pi$-Systeme. Die $\pi$-Wechselwirkung zweier Radikale (iv) setzt die Disproportionierungsstabilität der radikalischen Spezies voraus. Auch die Lage des $\pi$ Komplexbildungsgleichgewichtes dürfte hier von Bedeutung sein. Komplexe mit gemischte Redoxstufen $(v)$ sind vollständigkeitshalber aufgeführt und spielen bei der NIR-Absorption von Küpenfarbstoffen keine Rolle.

\section{Chemische Natur des NIR-Chromo- phors}

Aufgund dieser Analyse und unter Berücksichtigung, dass der Fürbeprozess eine chemische Reduktion und Oxidation beinhaltet, sind wir von der Hypothese ausgegangen, dass die NIR-Absorption des untersuchten Farbstoffes radikalischen Ursprungs ist. Unter der Annahme, dass die Farbstoffmolekuile entweder als diamagnetische Spezies $(S=0)$ oder als freie Radikale $(S=1 / 2)$ vorliegen, ergaben daraufhin ESRAbschätzungen einen Radikalgehalt von etwa $5 \mathrm{~mol}-\%$ in den gefärbten Textilproben mit dem Farbstoff C.I. Vat Black 30, der begleitet ist von der bereits erwähnten NIRAbsorption. Eine Festkörperprobe des Farbstoffes vor der Applikation zeigt keinen nennenswerten Paramagnetismus und keine NIR-Absorption im KBr-Pressling. Eine weitere Farbstoffprobe, die zuvor einem, dem Färbeprozess nachgeahmten Reduktions-Oxidations-Zyklus unterworfen («umgeküpt») wurde, war ebenfalls paramagnetisch, mit einem geschätzten Radikalgehalt von etwa $6 \mathrm{~mol}-\%$. Sie wies eine starke NIRAbsorption auf, welche mit jenen der Textilproben vergleichbar war. Statistisch gesehen bedeuten diese Daten, dass in den NIR-absorbierenden Textil- oder Festkörperproben dieses Farbstoffes etwa jedes 20. Farbstoffmolekül ein freies Radikal ist [9].

Schon 1945 beobachtete $H$. Hauser (Ciba), der Entdecker des C.I. Vat Black 30 , dass dieser Farbstoff durch Färben chemisch verändert wird. Dieser Befund ist im Einklang mit den spektralen Beobachtungen. Es hat sich im Laufe unserer Untersuchungen gezeigt, dass das 1,1'-Dianthrimid (DAI) ein Kernstrukturelement ist, welches noch alle wesentlichen Eigenschaften des ursprünglichen Farbstoffes zeigte. DAI liefert durch thermische Behandlung in $90 \%$ Schwefelsäure ein orangefarbenes Phthaloyl-ceramidon (PCO), dessen Struktur durch Elementaranalyse, HR-MS, ${ }^{13} \mathrm{C}-, 2 \mathrm{D}$ 'H-NMR- und Overhauser-Experimente bestätigt werden konnte. Es absorbiert nur im Sichtbarem (480 nm/ $\varepsilon=10300$ in Pyridin). Sowohl DAI wie PCO lassen sich durch $\mathrm{Na}_{2} \mathrm{~S}_{2} \mathrm{O}_{4} / \mathrm{OH}^{-}$und anschliessender LuftOxidation in ein tieffarbiges, schwerlösliches Produkt umwandeln, dem wir die Struktur $(\mathbf{P C O} / \mathbf{H}) \cdot$ zuschreiben. Letzteres entsteht aus PCO auch durch $\mathrm{Mg} / \mathrm{AcOH}$ Reduktion. (PCO/H) absorbiert stark im NIR (Fig. 2). Die beiden langwelligen Übergänge zeigen eine ausgeprägte vibronische Feinstruktur mit einer dominanten Frequenz $\left(\sim 1390 \mathrm{~cm}^{-1}\right)$ und sind einander in ihren Bandenformen sehr ähnlich. Den ersten Übergang im Lösungsmittel l-Cl-Naphthalin (CLN) interpretieren wir als ChargeTransfer-(CT)-Absorption des Dimeren ( $\pi$-Komplex im Sinne von Punkt (iv)) mit

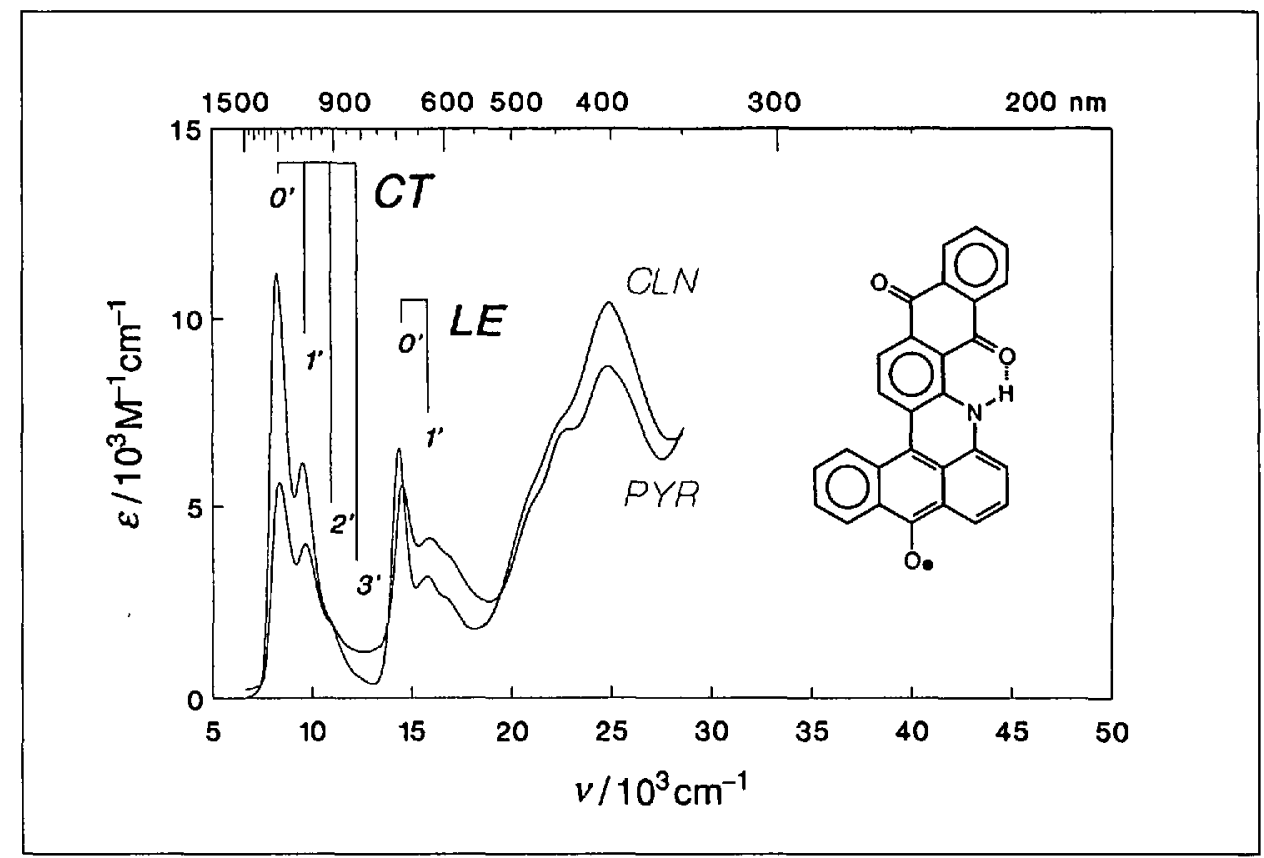

Fig. 2. Absorptionsspektren von (PCO/H) in 1-Chloronaphthalin (CLN) bei $90^{\circ}\left(7.96 \times 10^{-5} \mathrm{M}\right)$ und in Pyridin (PYR) bei RT. $\left(9.12 \times 10^{-5} \mathrm{M}\right)$ 
CHIMIA 45 (1991) Nr. 10 (Oklober)

Polarisationsrichtung senkrecht zur Molekülebene $\left(0^{\prime}, 0^{\prime \prime}\right.$-Bande: $1205 \mathrm{~nm} / \varepsilon=10800$; I',0"Bande: $1042 \mathrm{~nm} / \varepsilon=6370$ ) und den zweiten als lokale Anregung (LE) in der Molekülebene, welche sowohl in den Monomeren wie auch in der Dimerenstruktur aktiv sein kann $\left(0^{\prime}, 0^{\prime \prime}\right.$-Bande: $691 \mathrm{~nm} / \varepsilon=$ $6960 ; 1$ l',0"-Bande: $630 \mathrm{~nm} / \varepsilon=3690$ ). Diese Zuordnung ist ferner gestützt durch die Medienabhängigkeit der CT-Intensität, welche in Pyridin fast nur halb so gross ist $\left(0^{\prime}, 0 "\right.$-Bande: $1198 \mathrm{~nm} / \varepsilon=5650 ; 1^{\prime}, 0 "$. Bande: $1036 \mathrm{~nm} / \varepsilon=4^{\prime} 020$ ) wie in CLN, während die anderen Banden nur wenig beeinflusst werden.

Der Radikalgehalt von $(\mathrm{PCO} / \mathrm{H}) \cdot$ beträgt nach ESR-Messungen $12 \mathrm{~mol}-\% \mathrm{im}$ Festkörper. Da diese Probe wesentlich reiner ist, erscheint ein direkter Vergleich mit dem Radikalgehalt des C.I. Vat Black 30 nur der Grössenordnung nach zulässig. In CLN liefert $(\mathbf{P C O} / \mathrm{H}) \cdot$ ein ENDOR- und ein ESRSpektrum von grosser Komplexität (Fig. 3a). Beide sind mit den HMO-Spinpopulationen der angenommenen Radikalstruktur gut verträglich. Mit $\mathrm{CD}_{3} \mathrm{COOD}$ lässt sich das mobile NH-Proton austauschen. Das Spektrum zeigt dannach etwa 65 aufgelöste $\mathrm{Hy}$ perfeinlinien (Fig. 3b). Nach spektroelektrochemischen Versuchen entsteht $(\mathrm{PCO} / \mathrm{H})$ eindeutig in einem $1 \mathrm{e}^{-}$-Reduktionsprozess aus $\mathbf{P C O}$.

Damit ist die Natur der NIR-absorbierenden Spezie im Falle des einfachen 1,1'Dianthrimids geklärt: Im Färbeprozess bildet sich ein Phthaloyl-ceramidonyl-Radi$\mathrm{kal},(\mathbf{P C O} / \mathbf{H}) \cdot$, das im Gleichgewicht mit seinem $\pi$-Komplex steht. Letzterer beherrscht das Gleichgewicht und ist für die langwellige Absorption bei 1200 und 690 $\mathrm{nm}$ verantwortlich. Dieser $\pi$-Komplex ist kein Chinhydron, woraus sich auf grosse Disproportionierungsstabilität schliessen lässt. Die grosse Ähnlichkeit zwischen den
$(\mathrm{PCO} / \mathrm{H})-$ Spektren und den Spektraldaten des Farbstoffes auf Baumwolle zeigt, dass dieses Resultat zwanglos auf den Farbstoff C.I. Vat Black 30 übertragbar ist.

\section{Folgerungen}

In mehreren NIR-absorbierenden Küpenfarbstoffen sind 1,1'-Dianthrimidstrukturen entweder als Haupt- oder als Nebenkomponente enthalten oder treten in Zwischenprodukten auf. Eine eventuelle Säurebehandlung und/oder eine Reduktion im Färbeprozess, kann dann im Prinzip zu ähnlichen freien Radikalen mit NIR-absorbierenden $\pi$-Komplexen führen. Bei anderen, in reiner Form diamagnetischen, Küpenfarbstoffen entstehen freie Radikale bereits in der Synthese als paramagnetische Verunreinigungen. Violanthron $(C . I$. Vat Blue 20) ist ein solches Beispiel. Nach unseren Messungen absorbieren seine Baumwollfärbungen stark im NIR. Aus dem Rohfarbstoff konnte Aoki ein Nebenprodukt isolieren [10], dessen Radikalgehalt nach ESR-Messungen auf 40 mol-\% geschätzt wurde [11]

Das wichtigste Ergebnis dieser Arbeit ist a) eine Hypothese, wonach die NIR-Absorption der Küpenfarbstoffe radikalischen Ursprungs ist und $b$ ) die Tatsache, dass in den untersuchten Fällen die Hypothese auch zutrifft. Im allgemeinen dürfte die Struktur der freien Radikale sehr vielfältig sein. Sie können sowohl Haupt- wie Nebenprodukte der Synthese oder des Färbeprozesses sein und im unterschiedlichen Ausmass $\pi$ Komplexe bilden. Bei der Sichtung von mehreren Hundert Farbstoffstruktur- und Spektraldaten trafen wir nirgends auf ein Widerspruch. Auch ist eine alternative Erklärung für die NIR-Absorption nicht in Sicht.

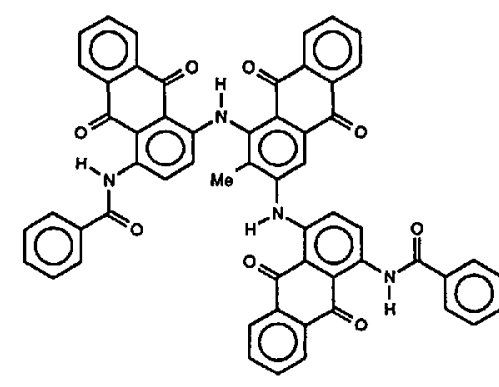

C.I. Vat Black 30

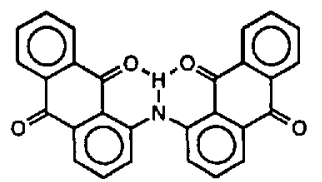

DAI

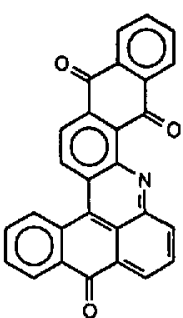

PCO

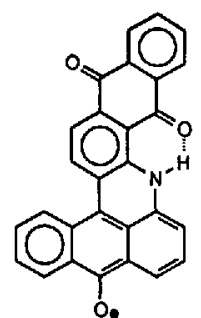

$(\mathrm{PCO} / \mathrm{H})^{\bullet}$

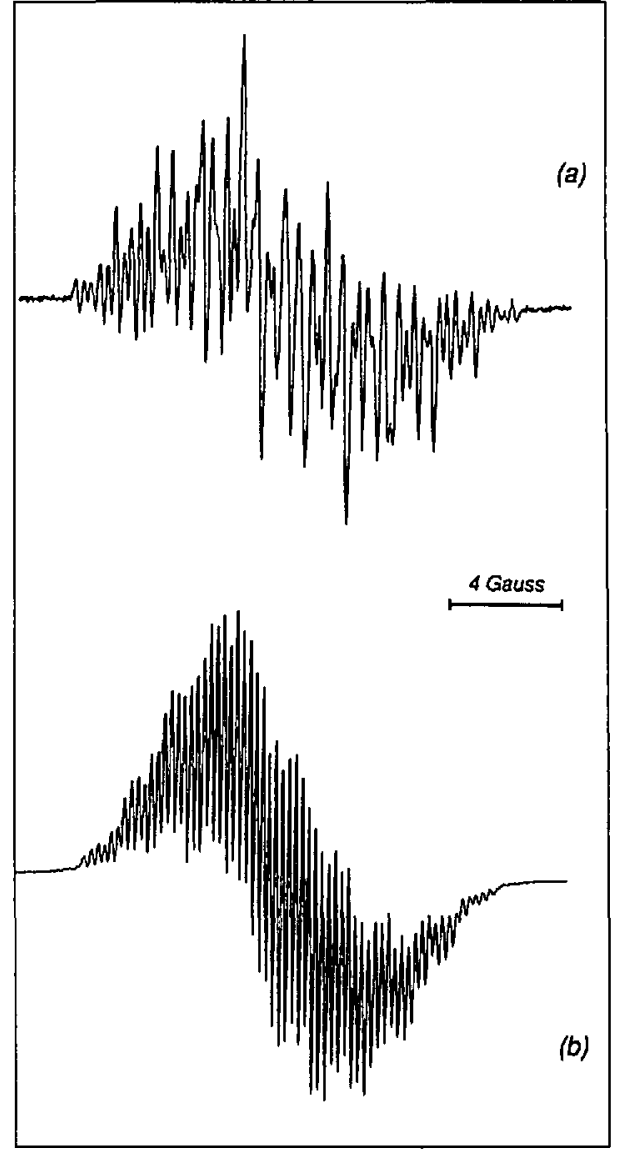

Fig. 3. ESR-Spektren von ( $\mathrm{PCO} / \mathrm{H}) \cdot$ in l-Chloronaphthalin a) ohne und b) mit Zusatz von $C D, C O O D$

[1] In Anlehnung an eine Idee vonJ.Piccard (1913) werden Farbstoffe, deren erster erlaubter Elektronenübergang ( Farbbande») jenseits der langwelligen Grenze des sichtbaren Spektralgebietes in NIR liegt und deren Farbe von höheren Elektronenzuständen bestimnt wird, als Farbstoffe zweiter Ordnung bezeichnet. Unter Farbstoffe erster Ordnung oder unter Farbstoffe schlechthin verstehen wir solche, mit Farbbanden im VIS.

[2] D.h. spektrale Verteilung der Quotienten aus Absorptions (K)- und Streu (S)-Koeffizienten nach Kubelka-Munk. Sie approximieren das Absorptionspektrum, sofern S nicht stark wellenlängenabhängig ist.

[3] J. Fabian, R. Zahradnik, Angew'.Chem. 1989, 101, 693.

[4] U. Wild, Chimia 1973, 27, 542.

[5] M.J.S. Dewar, H. Rogers, J.Am.Chem.Soc. 1962, 84, 395.

[6] Aus dichroitischen Messungen am Einkristall: K. Nakamoto,J.Am. Chen. Soc. 1952, 74, 1739.

[7] K. H. Hausser, J. N. Murrell,J.Chem. Phys. 1957, 27. 500 .

[8] D. Makovitsi, Th.-H.Tran-Thi, R. Even, J.Simon, Chem. Phys. Lett. 1987, 137, 107; A. deCian, M. Mousavi, J. Fischer, R. Weiss, Inorg. Chem. 1985, 24, 3162 .

[9] ESR-Messungen zeigen selbst 8-10 Jahren nach der Ausfärbung einen vergleichbaren Gehalt der Radikale in den Textilproben, was für die grosse Persistenz dieser Radikale spricht.

[10] J. Aoki, Bull. Chem. Soc. Jpn. 1961, 34, 1817.

[11] H. Akamatu, T. Maekawa, Y.Jido, M. Kinoshita, Bull.Chem.Soc.Jpn. 1964, 37, 849; vgl. auch K. Venkataraman, V. N. Iyer, in 'The Chemistry of Synthetic Dyes', Ed. K. Venkataraman, Academic Press, New York, 1971, Vol. 5, S. 204. 